\title{
Relationship between cardiovascular risk factors and traditional Chinese constitution in subjects with high-normal blood pressure
}

\author{
Hongju Jiang ${ }^{1}$, Youwei Han ${ }^{1}$, Runzhi Li $^{1}$, Suning Zhou ${ }^{2}$, Xiaoxia Liu ${ }^{1}$, Cong Cui ${ }^{1}$, Shouqiang Chen ${ }^{1}$, \\ Wei Zhang ${ }^{3^{*}}$ \\ ${ }^{1}$ Department of Cardiology, The Second Affiliated Hospital, Shandong University of Traditional Chinese Medicine, Jinan, China \\ ${ }^{2}$ Department of Cardiology, Provincial Hospital Affiliated to Shandong University, Jinan, China \\ ${ }^{3}$ Department of Cardiology, The First Affiliated Hospital, Shandong University of Traditional Chinese Medicine, Jinan, China \\ Email: jianghongju@medmail.com.cn
}

Received 14 January 2013; revised 13 March 2013; accepted 16 April 2013

Copyright (C) 2013 Hongju Jiang et al. This is an open access article distributed under the Creative Commons Attribution License, which permits unrestricted use, distribution, and reproduction in any medium, provided the original work is properly cited.

\begin{abstract}
Objective: We explored the relationship between traditional Chinese constitution and risk factors for cardiovascular disease in subjects with high-normal blood pressure (BP). Methods: The traditional Chinese constitution of 80 subjects with high-normal BP was judged by questionnaires and interviews. Measurements of cardiovascular and metabolic health included BP, body weight, body mass index (BMI), low-density lipoprotein cholesterol (LDL-C), 2-hour oral glucose tolerance test (OGTT2h), and waist circumference. Statistical relationships between cardiovascular disease risk factors and specific Chinese constitution were evaluated by univariate and multivariate analyses. Results: Ping He, Tan Shi, and Yin $\mathrm{Xu}$ were the dominant constitutions in subjects with high-normal blood pressure. There were significant differences in weight, LDL-C, BMI, OGTT2h, and waist circumference between Tan Shi and Ping He (P $<0.01$ ), in SBP and OGTT2h between Yin Xu and Ping He $(P<0.05)$, and weight, BMI, waist circumference, SBP, and LDL-C between Tan Shi and Yin $X u$ constitutions $(P<0.01)$. Logistic regression found a strong association between the Tan Shi constitution and LDL-C in subjects with high-normal blood pressure. Conclusion: Ping He, Tan Shi, and Yin Xu are the principle traditional Chinese constitutions in subject with high-normal blood pressure. The Tan Shi constitution is associated with multiple risk factors for cardiovascular disease (LDL-C, BMI, body weight, and waist circumference), while OGTT2h is closely related to the Yin $\mathrm{Xu}$ constitution.
\end{abstract}

${ }^{*}$ Corresponding author.
Keywords: High-Normal Blood Pressure; Traditional Chinese Constitution; Cardiovascular Risk Factors

\section{INTRODUCTION}

High-normal blood pressure (BP) puts patients at significantly greater risk for cardiovascular and cerebrovascular events. Both international and Chinese hypertension guidelines recommend therapeutic intervenetion for high-normal BP, especially when associated with other cardiovascular risk factors. Early interventional can prevent or significantly delay the development of cardiovascular and kidney diseases. Theories of traditional Chinese medicine emphasize good health practices and preventative therapies to reduce the incidence of cardiovascular and other diseases. We have identified susceptible populations according to the traditional Chinese constitution and developed therapies tailored for susceptible constitutions to reduce the incidence of disease and improve quality of life.

In the current study, we judged the traditional Chinese constitution of subjects with high-normal BP and explored the association between specific constitutions and cardiovascular risk factors to facilitate the development of preventive treatments for at-risk populations and to provide insight into the physiological correlates of traditional Chinese constitutions.

\section{MATERIALS AND METHODS}

\subsection{Subjects}

Eighty consecutive patients with high-normal BP (40\% males) who visited our hospital from April 2011 to November 2011 were enrolled in this study [1]. The inclu- 
sion criteria were as follows: outpatients of either sex, 18 to 65 years of age, with high-normal BP (diastolic blood pressure $[\mathrm{DBP}] \geq 85 \mathrm{mmHg}$ and $<90 \mathrm{mmHg}$, systolic blood pressure $[\mathrm{SBP}] \geq 130 \mathrm{mmHg}$ and $<140 \mathrm{mmHg}$ ). Patients with clinical hypertension and patients on antihypertensive medications, patients with liver, kidney, or hematopoietic system diseases, and patients with a history of stroke, myocardial infarction, diabetes, or malignnant tumors were excluded. Also, patients participating in drug clinical trials and those on long-term drug therapy were excluded from the study.

The 80 patients were divided into five groups according to their constitution: Ping He $(\mathrm{n}=32)$, Tan Shi $(\mathrm{n}=$ $30)$, Yin $\mathrm{Xu}(\mathrm{n}=16)$, Yang $\mathrm{Xu}(\mathrm{n}=1)$, and Te Bing $(\mathrm{n}=$ 1). The latter two constitutions were not analyzed statistically due to low numbers. Informed consent was obtained from all subjects based on a protocol approved by the Ethics Committee of the $2^{\text {nd }}$ affiliated Hospital of Shandong University of Traditional Chinese Medicine.

\subsection{Measurements}

Blood pressure, body weight, height, neck circumference, waist circumference, blood and urine chemistry, and other measurements were performed at the outpatient Clinic.

Blood pressure was measured using a mercury sphygmomanometer. The subjects were instructed to visit the clinic in a fasted condition between 8:30 and 11:30 a.m. After relaxing for 20 minutes or longer, blood pressure was measured three times on the left upper arm at one minute intervals with the patient in a sitting position, each time measured by a different trained registered nurse. The same three nurses measured BP for all patients. The median systolic blood pressure value was counted as the blood pressure for that day.

The neck circumference was measured at the connection between the margo superior of the $7^{\text {th }}$ cervical vertebrae and the inferior border of the Adam's apple.

Waist circumference was measured in the standing position using a non-elastic flexible tape. The tape was applied horizontally between the lowest rib margin and the iliac crest. Hip circumference was measured at the maximal protrusion of the buttocks.

Weight and height were measured on all visits. Subjects wore a shirt/blouse and trousers/skirts but no jewelry. The subjects were requested to wear the same clothes for each body weight measurement. Body mass index (BMI) was calculated based on these weight and height measurements as weight $/$ height $^{2}$ in $\mathrm{kg} / \mathrm{m}^{2}$.

Blood samples were taken from each subject following an overnight fast. The biochemical markers measured were glucose, total cholesterol (TC), LDL cholesterol (LDL-C), HDL cholesterol (HDL-C), triglyceride (TG), glutamic oxaloacetic transaminase, glutamic pyruvic trans- aminase, $\gamma$-glutamyltranspeptidase, lactate dehydrogenase, total protein, blood urea nitrogen, uric acid, creatine, sodium, potassium, and calcium. The urinalysis was carried out using appropriate test paper at the clinic.

All the subjects were placed on a diet consisting of at least $250 \mathrm{~g}$ carbohydrates per day for 3 days before testing blood and urine testing. After an overnight fast, a 2-h oral glucose tolerance test (OGTT) was performed at 9:00 AM with $75 \mathrm{~g}$ of glucose diluted in $200 \mathrm{ml}$ of water. Morning urine routines and urinary microalbuminuria were also measured.

\subsection{Questionnaires}

All subjects completed a High-Normal Blood Pressure Observation Table and a Traditional Chinese Constitution Classification and Identification Table. If the subject could not complete the classification and identification table, we judged constitution by interviews assessing quality of life and health.

\subsection{Statistical Analyses}

All measured values are expressed as mean \pm SD unless otherwise noted. Statistical analyses were carried out using SPSS 17.0 for Windows. All the continuous variables were tested for normal distribution. Group means were compared by ANOVA. Furthermore, we used Bartlett test to analyze the homoscedasticity of the variables. Significant variables associated with a particular traditional Chinese constitution by univariate analysis were entered into a multivariate regression model to identify independent predictors of traditional Chinese constitution. A $P<0.05$ (two tailed) test was considered statistically significant.

\section{RESULTS}

There were no significant differences in age, height, DBP, fasting glucose, TG, or TC among the Ping He, Tan Shi, and Yin $\mathrm{Xu}$ groups. Patients of Tan Shi constitution had significantly higher mean BMI, body weight, and waist circumference compared to the other two groups. Patients of Yin Xu constitution had lower mean systolic BP compared to the other two groups. The OGTT2h was significantly higher in the Tan Shi and Yin $\mathrm{Xu}$ groups than the Ping He group, whereas fasting glucose showed no difference among the three groups. Compared to the Ping He and Yin Xu groups, subjects of Tan Shi constitution had a significantly higher mean LDL-C (Table 1).

\section{MULTIVARIATE ANALYSIS OF PREDICTORS OF TRADITIONAL CHINESE CONSTITUTION}

Multivariate logistic regression analysis was performed 
Table 1. Clinical characteristics of the three traditional Chinese constitution groups.

\begin{tabular}{|c|c|c|c|}
\hline & Ping $\mathrm{He}(\mathrm{n}=32)$ & Tan Shi $(\mathrm{n}=30)$ & Yin $\mathrm{Xu}(\mathrm{n}=16)$ \\
\hline Age (years) & $54.91 \pm 4.47$ & $53.42 \pm 2.64$ & $50.25 \pm 3.00$ \\
\hline BMI $\left(\mathrm{kg} / \mathrm{m}^{2}\right)$ & $24.05 \pm 1.97$ & $28.78 \pm 1.76^{\star \#}$ & $23.97 \pm 2.21$ \\
\hline Weight (kg) & $70.65 \pm 6.77$ & $85.25 \pm 6.24^{\star \#}$ & $69.44 \pm 9.60$ \\
\hline Height $(\mathrm{cm})$ & $171.41 \pm 5.38$ & $172.07 \pm 4.74$ & $169.94 \pm 7.23$ \\
\hline Waist circumference $(\mathrm{cm})$ & $89.91 \pm 6.55$ & $99.30 \pm 6.34^{\star \#}$ & $88.63 \pm 7.25$ \\
\hline $\mathrm{SBP}(\mathrm{mmHg})$ & $130.31 \pm 5.64$ & $130.80 \pm 5.42$ & $125.63 \pm 7.08^{8^{*}}$ \\
\hline $\mathrm{DBP}(\mathrm{mmHg})$ & $85.91 \pm 2.70$ & $85.63 \pm 2.68$ & $86.38 \pm 1.75$ \\
\hline fasting glucose $(\mathrm{mmol} / \mathrm{L})$ & $4.67 \pm 0.60$ & $4.93 \pm 0.68$ & $4.54 \pm 0.77$ \\
\hline OGTT2h (mmol/L) & $6.15 \pm 1.60$ & $7.18 \pm 1.93^{\S}$ & $7.46 \pm 1.62^{\S}$ \\
\hline $\mathrm{TG}(\mathrm{mmol} / \mathrm{L})$ & $1.47 \pm 0.92$ & $1.71 \pm 0.72$ & $1.49 \pm 0.94$ \\
\hline $\mathrm{TC}(\mathrm{mmol} / \mathrm{L})$ & $5.34 \pm 0.87$ & $5.59 \pm 1.02$ & $5.18 \pm 0.81$ \\
\hline LDL-C (mmol/L) & $2.77 \pm 0.71$ & $3.31 \pm 0.65^{\star *}$ & $2.85 \pm 0.52$ \\
\hline
\end{tabular}

BMI: body mass index; SBP: systolic blood pressure; DBP: diastolic blood pressure; TG: triglyceride; TC: total cholesterol; LDL-C: low density lipoprotein-cholesterol; ${ }^{\star} P<0.01$, compared to Ping He constitution group; ${ }^{\sharp} P<0.01$, compared to Yin Xu constitution group; ${ }^{\S} P<0.05$, compared to Ping He constitution group; ${ }^{\star *} P<0.05$, compared toYin Xu constitution group.

to investigate the associations between traditional Chinese constitution and cardiovascular risk factors in the Tan Shi (Table 2) and Yin Xu groups (Table 3). The LDL-C was a significant predictor of Tan Shi constitution $(P=0.013)$ in subjects with high-normal blood pressure, while none of the entered variables was an independent predictor of the Tin Xu constitution $(P>0.5)$.

\section{DISCUSSION}

The concept of prehypertension was first proposed by the National Institute of Health of the United States in report JNC-7 [2]. Prehypertension refers to systolic blood pressure (BP) of 120 - $139 \mathrm{mmHg}$ and (or) diastolic BP of 80 $89 \mathrm{mmHg}$, an intermediate state between normal BP and hypertension. Normal BP according to guidelines for hypertension prevention and treatment by the Chinese Hypertension League (revised in 2010) is systolic BP of 120 $139 \mathrm{mmHg}$ and (or) diastolic BP of $80-89 \mathrm{mmHg}$, which is equivalent to prehypertension as proposed in JNC-7. The incidences of obesity and blood glucose abnormalities was significantly higher in patients with high-normal BP than in the ideal BP group [3]. Moreover, changes to both cardiovascular structural and function are observed in subjects with high-normal BP, including greater arterial intima-media thickness, reduced arterial elasticity, left ventricular hypertrophy, and diastolic dysfunction $[4,5]$. These studies emphasize the importance of timely screening of cardiovascular risk factors and inter- vention in subjects with high normal BP.

Traditional Chinese constitution can be classified as
Ping He, Tan Shi, Shi Re, Yin Xu, Yang Xu ,Qi Xu, Xue Yu, Qi Yu, or Te Bing. The specific traditional Chinese constitution may play an important role in pathogenesis. Each constitution has distinct physical and psychological reactions, symptoms, adaptive responses, and tendencies. In the current study, the high-normal BP group included 32 cases of Ping He, 30 cases of Tan Shi, and 16 cases of

Table 2. Logistic regression results of Tan Shi constitution group.

\begin{tabular}{lccc}
\hline & OR & $P$ & $95 \%$ CI \\
\hline Weight & 1.141 & 0.505 & $-0.105-0.210$ \\
BMI & 0.975 & 0.846 & $-0.504-0.415$ \\
Waist circumference & 1.004 & 0.363 & $-0.009-0.024$ \\
FBG & 1.127 & 0.679 & $-0.162-0.107$ \\
OGTT2h & 0.818 & 0.186 & $-0.015-0.076$ \\
TC & 0.946 & 0.799 & $-0.146-0.113$ \\
TG & 1.143 & 0.366 & $-0.164-0.062$ \\
LDL-C & 1.982 & 0.013 & $0.044-0.350$ \\
Smoking & 0.923 & 0.692 & $-0.214-0.143$ \\
Drinking & 1.037 & 0.735 & $-0.167-0.235$ \\
Campaign & 1.121 & 0.406 & $-0.258-0.106$ \\
Salt intake & 1.079 & 0.298 & $-0.064-0.206$ \\
\hline
\end{tabular}

OR: odds ratio; $\mathrm{CI}$ : confidence interval; BMI: body mass index; FBG fasting blood glucose; PBG: postprandial blood glucose; TG: triglyceride; TC: total cholesterol; LDL-C: low density lipoprotein-cholesterol. 
Table 3. Logistic regression results of Yin $\mathrm{Xu}$ constitution group.

\begin{tabular}{llll}
\hline & OR & $P$ & $95 \%$ CI \\
\hline Weight & 1.037 & 0.310 & $-0.181-0.552$ \\
Body mass index & 1.083 & 0.310 & $-1.588-0.521$ \\
Waist circumference & 1.025 & 0.533 & $-0.034-0.018$ \\
FBG & 1.056 & 0.903 & $-0.207-0.234$ \\
OGTT2h & 0.974 & 0.071 & $-0.007-0.152$ \\
TC & 1.325 & 0.542 & $-0.279-0.149$ \\
TG & 1.147 & 0.505 & $-0.236-0.119$ \\
LDL-C & 1.087 & 0.596 & $-0.193-0.330$ \\
Smoking & 1.112 & 0.115 & $-0.576-0.066$ \\
Drinking & 0.829 & 0.109 & $-0.062-0.584$ \\
Campaign & 1.185 & 0.519 & $-0.201-0.389$ \\
Salt intake & 0.936 & 0.765 & $-0.248-0.184$ \\
\hline
\end{tabular}

OR: odds ratio; CI: confidence interval; BMI: body mass index; FBG: fasting blood glucose; PBG: postprandial blood glucose; TG: triglyceride; TC: total cholesterol; LDL-C: low density lipoprotein-cholesterol.

Yin Xu constitution, but only one case each of Yang Xu and Te Bing constitution. Based on this sample, subjects of Tan Shi or Yin Xu constitution are more likely to have normal-high BP. Moreover, these three major constitution groups exhibited differences in several metrics of cardiovascular disease risk, including BMI, weight, waist circumference, SBP, and LDL-C. General features of the Tan Shi ("damp phlegm") constitution include obesity, abdominal fatness, and sticky greasy tongue presentation. General features of the Yin Xu ("shortage of Yin fluid") constitution include dry mouth, a slim figure, and "handfoot-heart hot" and other virtual thermal performances. People with the Ping He constitution usually have harmonized yin-yang and qi-blood. The main features of the Ping $\mathrm{He}$ constitution are a well-formed figure, ruddy color, and abundant energy. Subjects with Tan Shi constitutions had greater mean weight and waist circumference than subjects with Ping He or Yin Xu constitutions.

A comparison of 23 cases of obesity with Tan Shi constitution and 21 cases of obesity without Tan Shi constitution [6] revealed significantly higher TC, TG, LDL-C, blood glucose, and insulin in subjects with the Tan Shi constitution. The current study found significantly higher postprandial blood glucose and LDL-C in subjects with the Tan Shi and Yin Xu constitutions compared to the Ping He constitution, suggesting that subjects of Tan Shi and $\mathrm{Yin} \mathrm{Xu}$ constitution with high-normal BP also have lipid metabolism disorders and so may be at significantly greater risk of cardiovascular disease. Survey data has associated the Tan Shi constitution with a history of life- style diseases, less exercise, obesity, smoking, high BP, longer sleep duration and irregular sleep, and high-fat diets [7].

Many chronic diseases such as diabetes, hyperthyroidism, hypertension, chronic kidney disease, and menopausal syndrome have pathogenic mechanisms and risk factors consistent with various physical and behavioral aspects of the Yin $\mathrm{Xu}$ constitution. High-normal BP is one specific aspect of subhealth, characterized by chronic fatigue and visceral disorders. Many of subjects with high-normal BP have the Yin Xu constitution, but the systolic BP of our Yin Xu sample was actually lower than that of Tan Shi and Ping He subjects $(P<0.05)$. Considering that the mean weight $\mathrm{Yin} \mathrm{Xu}$ subjects was lower and the number of subjects limited, further studies are needed to clarify the incidence of high-normal BP and hypertension in this group.

This research demonstrates a significant relationship between cardiovascular risk factors, such as high BMI, elevated blood lipids, higher postprandial blood sugar, and the Tan Shi constitution (particularly between LDL$\mathrm{C}$ and Tan Shi constitution). In addition, the sample suggests that Tan Shi and Yin Shi are the dominant constitutions of patients with high-normal BP. Thus, patients of these constitutions should be identified, evaluated, and treated as soon as possible to reduce the risk of cardiovascular disease. Large-scale clinical studies investigating the relationships between traditional Chinese constitution and disease risks are warranted.

\section{REFERENCES}

[1] Hypertension Prevention and Control Guidelines Revision Committee in China (2011) Prevention and treatment of hypertension in China guide 2010. Chinese Journal of Hypertension, 19, 701-743.

[2] Chobanian, A.V., Bakris, G.L., Black, H.R., et al. (2003) The 7 th report of the joint national committee on prevention, detection, evaluation and treatment of high blood pressure: The JNC 7 report. The Journal of the American Medical Association, 289, 2560-2572. doi:10.1001/jama.289.19.2560

[3] Kokubo, Y., Kamide, K., Okamura, T., et al. (2009) High normal blood pressure and the risk of cardiovascular disease. Circulation Journal, 73, 1381-1385. doi:10.1253/circj.CJ-09-0336

[4] Seong, H.K., Goo-Yeong, C., Inkyung, B., et al. (2011) Early abnormalities of cardiovascular structure and function in middle-aged Korean adults with prehypertension: The Korean genome epidemiology study. American Journal of Hypertension, 24, 218-224. doi:10.1038/ajh.2010.213

[5] Drukteinis, J.S., Roman, M.J., Fabsitz, R.R., et al. (2007) Cardiac and systemic hemodynamic characteristics of hypertension and prehypertension in adolescents and young adults: The strong heart study. Circulation Journal, 115, 
221-227.

[6] Su, Q.M. and Wang, Q. (1995) Bulk of phlegm-dampness constitution blood lipid, blood glucose, insulin and erythrocyte $\mathrm{Na}^{+}-\mathrm{K}^{+}$-ATP detection and characterization of enzyme activity. Journal of Basic Medical Science of Tra- ditional Chinese Medicine, 1, 39-41.

[7] Wang, Q. and Zhu, Y.B. (2008) Study on effect factors of traditional Chinese phlegm-dampness constitution. Journal of Beijing University of Traditional Chinese Medicine, 31, 10-13. 